\title{
Metodologías de aprendizaje cooperativo a través de Tecnologías de la Información y la Comunicación (TIC).
}

\section{Cooperative learning methodologies through Information and Communications Technology (ICT).}

\author{
Pedro P. Chamorro Barrancoํ, Bárbara Luque Salas², Amalia Reina Giménez³, \\ Diego García Peinazo ${ }^{4}$, David Ojeda Nogales ${ }^{5}$, Carmen de la Mata Agudo ${ }^{6}$, \\ Mónica Calderón Santiago7, David Gutiérrez Rubio ${ }^{8}$, \& Adoración Antolí \\ Cabrera ${ }^{9}$,
}

Fecha de recepción: 17/04/2020; Fecha de revisión: 23/05/2020; Fecha de aceptación: 01/06/20

Cómo citar este artículo:

Chamorro, P.P, Luque, B., Reina, A., García-Peinazo, D., Ojeda, D., De la Mata, C., Calderón-Santiago, M. Gutiérrez-Rubio, D \& Antolí, A. (2020). Desarrollo de metodologías de aprendizaje cooperativo a través de Tecnologías de la Información y la Comunicación (TIC). Revista de Innovación y Buenas Prácticas Docentes, 9(2), 1-16.

Autor de Correspondencia: pedro.chamorro@uco.es

\section{Resumen:}

El resumen contará con un máximo de 200 palabras.

El desarrollo de las tecnologías de la información y comunicación (TIC) permite en el ámbito docente la posibilidad de realizar actividades que suelen resultar atractivas para el alumnado. Cuando están vinculadas de una manera pertinente hacia las competencias que se pretenden conseguir en el proceso de enseñanza-aprendizaje, tienen un gran valor didáctico. Si este tipo de actividades se combina o enmarca en una metodología de trabajo cooperativo, el efecto puede ser muy positivo en la actividad docente. Las estructuras cooperativas gozan de un consistente apoyo en relación a los resultados de aprendizaje, así como sobre otros factores como la motivación personal, la valoración propia o las relaciones establecidas entre el alumnado. El presente trabajo describe una experiencia conjunta que pone en valor el trabajo cooperativo como estrategia didáctica, y la utilización de las TIC como instrumento metodológico en diferentes asignaturas y grados en la Universidad de Córdoba realizada durante el curso académico 2018-19. Se analizan sus efectos, así como aspectos a tener en cuenta para su implementación. Siendo la valoración general positiva se observa que el alumnado se implica y aumenta el trabajo autónomo con esta metodología junto con la necesidad de sistematización de la tarea por parte del profesorado.

\footnotetext{
I Universidad de Córdoba (España), pedro.chamorro@uco.es; CÓDIGO ORCID: 0000-0002-0940-2853

2 Universidad de Córdoba (España), bluque@uco.es; CÓDIGO ORCID: 000-0001-6858-9892

3 Universidad de Córdoba (España), amalia.reina@uco.es; CÓDIGO ORCID: 0000-0003-3916-9017

${ }_{4}$ Universidad de Córdoba (España), diego.garcia@uco.es; CÓDIGO ORCID: 0000-0002-0957-2828

5 Universidad de Córdoba (España), dojeda@uco.es; CÓDIGO ORCID: 0000-0001-5379-8069

- Universidad de Córdoba (España), m02maagm@uco.es; CÓDIGO ORCID: 0000-0001-5096-3399

7 Universidad de Córdoba (España), b42casam@uco.es; CÓDIGO ORCID: 000-0002-2044-2397

8 Universidad de Córdoba (España), dgrubio@uco.es; CÓDIGO ORCID: 0000-0002-4461-2223

9 Universidad de Córdoba (España), aantoli@uco.es; CÓDIGO ORCID: 0000-0001-7259-5080
} 
Palabras clave: Método de aprendizaje, TIC, proceso de interacción educativa, enseñanza en equipo.

\begin{abstract}
:
The development of the information and communication technologies (ICT) allows in the teaching field the possibility of performing activities which are attractive for the students. These have a considerable educational value when they are linked in a proper way with the skills intended to be reached in the teaching-learning process. If these types of activities are combined within a collaborative methodology frame, the impact could be highly positive in teaching. The cooperative layouts have a strong support related with the learning results, as well as other factors like personal motivation, a better self-esteem or the established relationships among students. The current work describes a joint experience that points out the value of cooperative learning as didactic strategy, and the use of ICT as a methodological instrument in different subjects and degrees at the University of Cordoba during the academic year 201819. It analyzes its effects, in addition to important matters for its implementation. Evaluating this experience in a positive way, it is observed student body is implied, their autonomous work grows together with the necessity of systematizing of the task by the professor.
\end{abstract}

Key Words: Learning methods, ICT, educational interaction process, team teaching.

Este trabajo recoge una experiencia de innovación realizada durante el curso 2018-19 en asignaturas de diferentes Grados de la Universidad de Córdoba (UCO). Se centra en el uso del aprendizaje cooperativo en el aula mediante la utilización de diversos recursos propios de las nuevas tecnologías de la información y la comunicación (TIC). La experiencia es realizada en el marco del Plan de Innovación Docente del mismo curso de la UCO y, en concreto, dentro de la modalidad relativa a la Formación en Innovación Docente. En el presente apartado se desarrolla el marco que sirve como introducción de la experiencia planteada, abordando en los siguientes epígrafes el desarrollo, resultados y conclusiones principales de la misma.

Una competencia básica demandada en el mercado laboral actual y con una clara perspectiva futura es la capacidad de trabajar en equipo, la cual viene potenciándose desde distintos ámbitos educativos. La utilización del aprendizaje cooperativo se configura como una metodología capaz de conseguir resultados óptimos para el proceso de aprendizaje, y también como instrumento para el entrenamiento y ensayo de estas habilidades y destrezas.

El trabajo cooperativo, como metodología, está ampliamente fundamentado en investigaciones psicopedagógicas (Fernández Berrocal y Melero, 1995; Colomina y Onrubia, 2001; Palomares-Montero y Chisvert-Tarazona, 2016) que exponen los beneficios derivados de esta práctica, señalando una mejora tanto en los resultados académicos como en las habilidades socioemocionales. Apoyados en la literatura científica y teniendo como referencia la convergencia con el interés que marca el Espacio Europeo de Educación Superior en las nuevas metodologías docentes, se fundamenta este trabajo.

En el caso de esta experiencia, las distintas actividades realizadas han combinado la aplicación de metodologías cooperativas con la utilización de las TIC, a las que el alumnado suele mostrar receptividad (Herrada y Baños, 2018). Estas están adquiriendo un rol central en los ámbitos de nuestra vida personal, laboral y social, así como en el ámbito de la educación, y permiten la realización de actividades que hace algún tiempo eran impensables. Las TIC favorecen un cambio de dirección de la enseñanza: de la primacía de la acumulación de datos, a un nuevo estado en el que se prioriza la importancia de su obtención, comprensión, uso e interpretación.

Trabajo cooperativo y utilización de TIC son dos elementos que favorecen el desarrollo de la motivación por parte del alumnado, aspecto clave en el aprendizaje. La motivación y su relación con la toma de conciencia por parte del alumnado de la relevancia de la tarea planteada para su aprendizaje suponen el desarrollo de 
habilidades metacognitivas (Ortega y Sánchez-Queija, 2014), habilidades que son las que han pretendido desarrollar. La movilización y combinación de procesos cognitivos, motivacionales y de autorregulación es lo que favorece la motivación interna o intrínseca del propio individuo hacia el aprendizaje.

Las actividades realizadas, desarrolladas en el siguiente apartado, se llevaron a cabo en titulaciones de las áreas de Ciencias de la Educación, y Arte y Humanidades. En ambos casos, se ha considerado que el trabajo cooperativo con utilización de las TIC es consecuente con la adquisición y desarrollo de competencias para la creación de entornos de trabajo colaborativos y redes de apoyo profesionales que permitan la gestión compartida del conocimiento.

\section{DESARROLLO DE LA EXPERIENCIA DE INNOVACIÓN}

La planificación y realización de las distintas actividades enmarcadas en esta experiencia fue realizada en dos momentos. Por una parte en el espacio y tiempo de cada asignatura, lo que nos permitió valorar el desarrollo de la experiencia. Y por otra parte, en una sesión final conjunta en el que los grupos participantes realizaron actividades de reflexión y abstracción sobre los contenidos aprendidos utilizando el método cooperativo, lo que ha permitido recoger datos sobre cómo han utilizado la tecnología como elemento de aprendizaje y valorar la combinación ambos elementos en la realización de las actividades.

A continuación, se indican (Tabla 1) las distintas asignaturas con las que los docentes participaron en el proyecto y la titulación a la que corresponden.

Tabla 1.

Asignaturas, curso y titulación con actividades realizadas en la experiencia innovadora.

\begin{tabular}{|c|c|c|c|}
\hline & Denominación asignatura & Curso & Titulación \\
\hline 1. & $\begin{array}{l}\text { Didáctica de las Ciencias Experimentales en } \\
\text { Educación Primaria }\end{array}$ & $3^{\circ}$ & $\begin{array}{l}\text { Grado en Educación } \\
\text { Primaria }\end{array}$ \\
\hline 2. & $\begin{array}{l}\text { Didáctica del Medio Ambiente en Educación } \\
\text { Infantil }\end{array}$ & $3^{0}$ & $\begin{array}{l}\text { Grado en Educación } \\
\text { Infantil }\end{array}$ \\
\hline 3. & Matemáticas & 10 & $\begin{array}{l}\text { Grado en Educación } \\
\text { Primaria }\end{array}$ \\
\hline 4. & Historia de la Música & $3^{\circ}$ & $\begin{array}{l}\text { Grado en Historia del } \\
\text { Arte } \\
\text { Grado en Gestión } \\
\text { Cultural } \\
\text { Doble Grado de } \\
\text { Historia e Historia del } \\
\text { Arte }\end{array}$ \\
\hline 5. & Arqueología del Mundo Clásico & $4^{\circ}$ & Grado en Historia \\
\hline 6. & Psicología de la Educación y el Desarrollo & 10 & $\begin{array}{l}\text { Grado en Educación } \\
\text { Primaria }\end{array}$ \\
\hline 7. & $\begin{array}{l}\text { Observación Sistemática en el Aula de } \\
\text { Educación Infantil }\end{array}$ & 10 & $\begin{array}{l}\text { Grado en Educación } \\
\text { Infantil }\end{array}$ \\
\hline
\end{tabular}

Fuente: Elaboración propia. 


\subsection{Descripción de las experiencias de trabajo cooperativo organizadas en cada asignatura.}

A continuación, se detallan las experiencias y su planteamiento.

\subsubsection{Didáctica de las Ciencias Experimentales en Educación Primaria (3ำ curso, Grado en Educación Primaria)}

Dentro de esta asignatura, el alumnado tuvo que preparar en pequeños grupos un tablón de anuncios virtual mediante la plataforma web "Padlet" sobre la deriva continental para que, de esta forma, adquiriera de forma cooperativa conocimientos acerca del tiempo geológico y la evolución de la Tierra. En este caso cada grupo fue encargado de desarrollar un tablón independiente, y dentro de cada grupo se asignaron distintas tareas: búsqueda de la fauna y flora de cada una de las eras geológicas, así como la estructuración de los continentes que la caracteriza. De esta forma, tuvieron que combinar toda la información al final, teniendo la visión completa del desarrollo histórico de nuestro planeta.

El uso de las TIC a través de la herramienta web "Padlet" permitió crear un mural virtual con información (texto, imágenes y vídeos) al que se pudo acceder a través de un enlace web. En este caso, usaron imágenes y descripciones para la flora y la fauna, pero para la estructuración de los continentes se les proporcionó cartulina y recortes, realizando ellos la composición y subiendo la fotografía al enlace web. Con esta experiencia no solo se profundizó en contenidos relevantes del currículum de Ciencias Naturales en Educación Primaria, sino que además se desarrollaron competencias necesarias para su transposición didáctica.

\subsubsection{Didáctica del Medio Ambiente en Educación Infantil (3ํㅡrado en Educación Infantil)}

Dentro de esta asignatura se desarrolló un proyecto final de forma cooperativa entre toda la clase. El proyecto final consistió en la preparación y desarrollo de una sesión educativa destinada a alumnado de Educación Infantil en un espacio lúdico al aire libre como es la Ciudad de los Niños y las Niñas de Córdoba. Para trabajar de forma cooperativa se dividió la clase en varios grupos de 4-5 integrantes cada uno de ellos y se le asignaron distintas tareas a cada uno de los grupos.

En primer lugar, cada grupo fue responsable de proponer una serie de actividades para ese día enfocadas a enseñar distintos problemas ambientales. Cada grupo expuso su propuesta al resto de clase y se hizo una selección de actividades para el proyecto final. A continuación, se dividieron las tareas por los grupos y entre todos, siguiendo interdependencia positiva tanto de materiales como de objetivos, se encargaron de preparar guiones para cada una de las actividades, organizar la sucesión de actividades a lo largo de la jornada, preparar los materiales necesarios para su desarrollo y repartirse las distintas tareas para que esa jornada. A través de este proyecto tuvieron que profundizar en las bases teóricas de la asignatura para poder proponer una transposición adecuada de cada uno de los problemas ambientales que conocían, y adquirir las destrezas necesarias para poder realizar una enseñanza significativa en el ámbito de la Educación ambiental y el desarrollo sostenible. 


\subsubsection{Matemáticas (1ำ curso, Grado en Educación Primaria)}

Se organizó una actividad por grupos de 4 personas en las que cada grupo tenía que desarrollar una parte del temario de la asignatura, indicando conceptos, problemas y biografía de algún matemático relacionado con el tema. Los contenidos se crearon en formato "Wiki colaborativo", alojado en ENOA 3 (un espacio virtual de la UCO para almacenar contenidos), de manera que estuvieran disponibles desde el comienzo para todo el alumnado. Cada miembro del grupo eligió el rol que debían tener dentro del grupo (redactar Conceptos, Problemas I, Problemas II y Biografía). La tarea de cada miembro del grupo consistió en, aparte de su rol específico (buscar problemas, una biografía, etc.) en corregir y revisar las aportaciones de los otros miembros del grupo. El carácter abierto de la "Wiki" implica que el contenido que iba subiendo cada grupo estaba inmediatamente visible al resto de la clase, pudiendo realizar comentarios de retroalimentación de forma continua durante el desarrollo de la actividad. Por su parte, la evaluación se realizó mediante dos rúbricas, una de carácter grupal (única) y otra individual (de 3 tipos diferentes, según el rol asignado).

\subsubsection{Historia de la Música ( $3^{\circ}$ curso, Grado en Historia del Arte; $3^{\circ}$ curso, Grado en Gestión Cultural; Doble Grado de Historia e Historia del Arte).}

La experiencia consistió en profundizar en los contenidos y competencias profesionales asociadas de la asignatura "Historia de la Música" y a los Grados mencionados a través de la elaboración cooperativa de un podcast. El alumnado, vinculado a la rama de Artes y Humanidades, pudo, por medio de este recurso, realizar un proyecto creativo y motivador en el cual se atendió de manera prioritaria a la divulgación cultural como actividad destacada como futuros profesionales de la cultura.

La misma se llevó a cabo mediante una metodología de aprendizaje basado en el análisis y diagnóstico de fuentes documentales -fundamentalmente, festivales preexistentes sobre música y exposiciones preexisten sobre música-. De esta forma, se planteó una metodología cooperativa a través del reparto de roles para la elaboración del podcast y estrategias de motivación basadas en la experimentación sonora y las TIC.

En un primer estadio, los grupos analizaron críticamente cuestiones de tipo histórico-musical y de la gestión del evento/exposición. Tras ello, elaboraron un guion radiofónico en el cual aparecen indicaciones explícitas sobre las intervenciones de cada uno de los miembros y, asimismo, una descripción de los fragmentos de obras musicales utilizadas. La tercera parte del proyecto consistió en la grabación y masterización del podcast, en el cual se comentan los aspectos más significativos de la exposición o el festival/ciclo analizado, empleando para ello diversos formatos radiofónicos -entrevista, concurso, etc.- Por último, el alumnado presentó sus propuestas al grupo clase.

\subsubsection{Arqueología del Mundo Clásico (4urso, Grado en Historia)}

Se dividió al alumnado en tres grupos y trabajaron sobre temas elegidos por ellos voluntariamente. Los bloques de trabajo fueron: "la evolución del templo griego", "la 
mitología clásica en los vasos griegos" y "el problema del frontón griego". Sobre cada uno de los temas el alumnado realizó un vídeo-documental.

El trabajo y los vídeos se realizaron en horario de clases. Para ello el profesorado actuó como tutor planteando preguntas que guían al alumnado y orientan la resolución de los problemas. De este modo, el alumnado va siendo guiado para resolver por sí mismo los problemas que plantea la Arqueología del Mundo Clásico.

Además del trabajo cooperativo y la utilización de las TIC, la metodología empleada en esta asignatura giró en torno a dos ejes. El primero es el Trabajo por Proyectos. Este modelo se basa en un trabajo educativo que generalmente se realiza durante un periodo de tiempo más o menos prolongado (La Cueva, 1998). Se caracteriza por una fuerte implicación del alumnado en su planteamiento, desarrollo y evaluación final. Es fundamental que un proyecto de este tipo combine la investigación empírica, con la reflexión acerca de la actividad realizada, la consulta bibliográfica y la comunicación de los resultados obtenidos.

El segundo es el Modelo Didáctico de Investigación en la Escuela. Este modelo busca la adecuación entre lo conceptual y lo procedimental, procurando solventar la antigua polémica y división entre ambos aspectos (García, 2000; García y García; 1989; Pineda y García, 2011). Se basa en el planteamiento de problemas en clase, su investigación en el aula y posterior resolución por parte del alumnado durante la secuencia de aprendizaje (Pineda y García, 2011).

\subsubsection{Psicología de la Educación y del Desarrollo (1ํurso, Grado en Educación Primaria)}

El alumnado se dividió en distintos grupos de trabajo (de 3-5 integrantes) según su elección y se realizaron distintas acciones. En primer lugar crearon un video en el que se incluían preguntas tipo cuestionario sobre algún modelo de desarrollo que se revisa en la asignatura o bien, la creación de un muro digital o pizarra colaborativa sobre los distintos mecanismos internos y externos de aprendizaje y que corresponden a otros temas de la asignatura. Para ello se utilizaron las aplicaciones Edpuzzle o Padlet. Tras esta tarea, todo el alumnado visualizó el material elaborado por grupos de su mismo grupo-clase, facilitando feedback a los grupos que lo habían creado mediante entradas en foros virtuales, el cual fue analizado por el grupo creador que estableció conclusiones sobre el mismo.

Además, el alumnado visualizó los productos del tipo de tarea que no han realizado y que ha sido realizada por grupos de un grupo-clase diferente, facilitándole igualmente feedback mediante entradas en foros virtuales creados para cada trabajo, el cual fue también analizado por los grupos creadores. Como actividad final, todo el alumnado cumplimentó un cuestionario sobre el valor otorgado al trabajo cooperativo realizado.

La metodología utilizada parte de la consideración de realización de un aprendizaje cooperativo, es decir un procedimiento donde un grupo de alumnado realiza, siguiendo cierta estructura, una actividad o tarea previamente determinada (Colomina y Onrubia, 2001). El procedimiento llevado a cabo fue:

1. Trabajo inicial de grupo: selección de contenidos a incluir y planificación de la realización de la actividad.

2. Puesta en común con el docente (recepción de feedback).

3. Desarrollo y realización de la tarea.

4. Presentación de los trabajos elaborados a los compañeros/as del mismo grupoclase. 
5. Visualización individual más específica de $\mathrm{n}^{\circ}$ limitado de presentaciones.

6. Remisión de feedback a los compañeros/as y recapitulación y análisis del mismo.

7. Repetición de las fases 5 y 6 con el trabajo elaborado por grupos de otros grupos-clase.

8. Por último, valoración del trabajo cooperativo.

\subsubsection{Observación Sistemática en el Aula de Educación Infantil (1ํㅜㄴ curso, Grado en Educación Infantil)}

La experiencia consistió en esbozar un trabajo de investigación en el que, tras plantear una pregunta de investigación, el alumnado debía elaborar tres instrumentos de observación sistemática. Para la realización de la tarea el alumnado se constituyó como grupo y acordaron unos principios sobre los que trabajar que se plasmaron en un contrato que han elaborado entre todas las personas integrantes del grupo. Las sesiones fueron orientadas con tareas consecuentes al objetivo final, cada una de ellas se inició repasando lo trabajado en la sesión anterior y planificando la tarea que realizar. El trabajo del grupo y las tareas individuales que cada persona se comprometió a aportar, se coordinaron a través de la figura de una persona responsable que hizo de relatora y compiladora de la tarea que se había realizado. EI resultado fue la exposición y la grabación en video de cada grupo exponiendo su trabajo de investigación.

Se utilizó un contrato de compromisos que el grupo previamente había negociado. Este documento sirve para que cada componente exprese su opinión acerca de los retos que se quieren obtener del trabajo en cooperación. Esto permite la negociación y el intercambio de opiniones de las personas del grupo. Por un lado, constituye una herramienta muy útil para que el propio alumnado autogestione su proceso de aprendizaje y su responsabilidad hacia la tarea. Por otro, incentiva y ejercita las habilidades de negociación y escucha.

Este contrato refleja los componentes del grupo y los roles asignados, las sesiones y las tareas que se realizan en cada una de ellas, junto con la valoración que se realiza durante la sesión y la evolución percibida respecto al aprendizaje. Esta aparece datada con fecha y con una relación de tareas que es necesario acometer en la sesión siguiente. También, el documento recoge lo que se ha realizado durante la sesión y lo qué se ha aprendido con respecto a la anterior. Para la edición de los videos se ha utilizado se ha utilizado la aplicación Inshot, una aplicación para la edición de vídeo y fotografía, orientada a mejorar el acabado de distintos vídeos realizados.

\subsection{Descripción de la actividad: I Muestra Virtual "El aprendizaje cooperativo en 59 segundos"}

Por último, y como cierre del proyecto para el alumnado se organizó la actividad denominada I Muestra Virtual "El Aprendizaje Cooperativo en 59 segundos" para fomentar la transferencia y el intercambio de conocimientos y experiencias del alumnado participante. Esta actividad se temporalizó dos semanas antes de la finalización del cuatrimestre y pretendía ser un espacio de encuentro (virtual) entre el 
alumnado de diferentes grados y asignaturas que permitiera implementar el valor de las TIC como instrumento pedagógico, a la vez que valorar cómo son utilizadas por el alumnado.

La participación, se planteó con carácter voluntario, y consistía en tres aspectos. De una parte, mostrar cada grupo en un vídeo de unos 59 segundos, alguna reflexión o aspecto sobre el trabajo cooperativo que se habría abordado. De otra parte, los participantes visionarían distintos vídeos de otros grupos y realizarían aportaciones en los diferentes foros sobre el trabajo de los compañeros.

La difusión de esta actividad se realizó de dos formas, de una parte en clase por parte del profesorado y de otra, en el espacio ENOA 3 en la plataforma Moodle de la UCO. Esta plataforma fue utilizada para el acceso a los contenidos de otros estudiantes y donde se realizaron las participaciones en los foros.

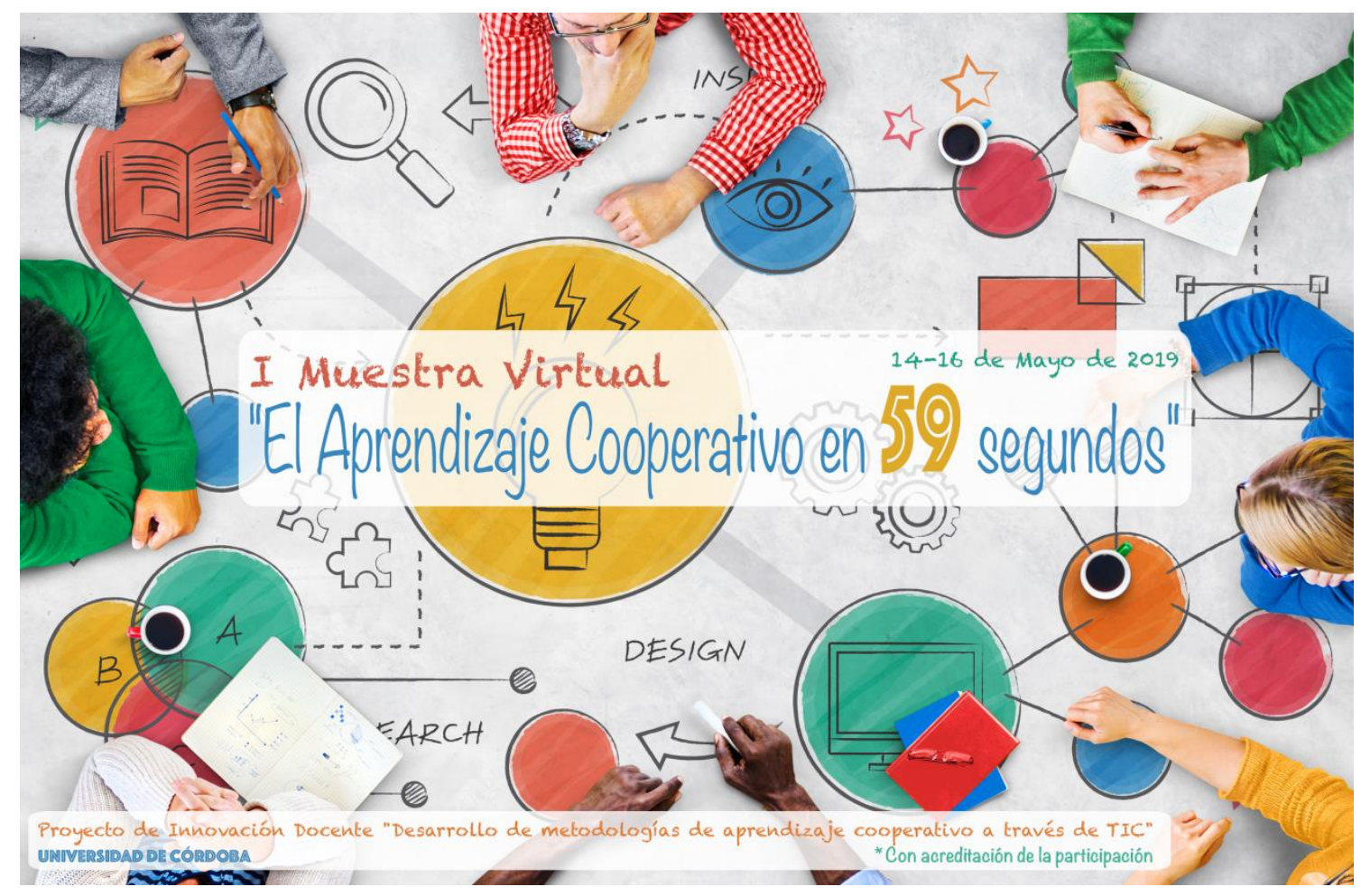

Figura 1. Cartel anunciador de la muestra.

\section{RESULTADOS}

Al igual que el apartado anterior, se detallan los resultados ofrecidos por la experiencia en cada una de las asignaturas. Y la actividad final, expresando también en este caso los principales resultados encontrados.

\subsubsection{Didáctica de las Ciencias Experimentales en Educación Primaria (3 Grado en Educación Primaria)}

Se obtuvieron resultados interesantes, pues muchos de los "Padlet" desarrollados por el alumnado estuvieron bien documentados y representados. Aunque otros tuvieron alguna carencia, en general los contenidos presentados fueron coherentes con la 
asignatura y demostraron haber adquirido un grado de conocimiento significativo acerca de la evolución del tiempo geológico. Todos los grupos sin excepción presentaron sus trabajos a tiempo. La evaluación de la eficiencia de esta herramienta y metodología de trabajo para alcanzar los objetivos de la asignatura la llevó a cabo el profesorado de la asignatura, mediante la corrección de los trabajos, todos con calificación superior a 7 sobre 10. Además, el trabajo se realizó de forma presencial en la asignatura, por lo que el profesorado pudo recoger testimonio visual de la eficacia de usar el aprendizaje cooperativo, a la par que sirvió de apoyo para dudas del alumnado.

\subsubsection{Didáctica del Medio Ambiente en Educación Infantil (3ํ Grado en Educación Infantil)}

El resultado en esta asignatura se valora como positivo, aunque algunos grupos no cumplieron a tiempo sus funciones. Como se puso de manifiesto en la sesión final que se dedicó a la reflexión sobre el trabajo realizado, todos los grupos constataron la importancia de la colaboración, no solo para el desarrollo óptimo de la actividad con el alumnado de infantil, sino también para la preparación de materiales y recogida de elementos reciclables, y se mostraron satisfechos con el resultado final. Se les pidió aportar su opinión sobre el proyecto realizado, y de viva voz todas comunicaron sus impresiones al docente. Como se ha mencionado el principal problema fue que algunos grupos no habían cumplido sus funciones, que tuvieron que ser realizadas por el resto de grupo. Era algo negativo, pero a su vez positivo, pues gracias a ello se involucraron en otras tareas y efectivamente constataron la importancia de que, en la metodología colaborativa, todos cumplan su parte.

\subsubsection{Matemáticas ( $1^{\circ}$ Grado en Educación Primaria)}

Como resultado más evidente de las actividades realizadas, se obtuvo un compendio de problemas, definiciones y biografías relacionadas con la asignatura, en formato digital, fácilmente accesible para el alumnado. Dicho material se utilizó por el conjunto del alumnado como apoyo para el estudio de la asignatura.

\subsubsection{Historia de la Música (3 Grado en Historia del Arte; 3 Grado en Gestión Cultural; Doble Grado en Historia e Historia del Arte):}

Las diferentes fases de la actividad contribuyeron significativamente a la adquisición de competencias profesionales vinculadas a los dos grados. En este sentido, la valoración fue positiva respecto a la implicación del alumnado y el nivel de ejecución relacionado con las competencias que se pretendían trabajar.

Entre los resultados, podemos destacar que la distribución de grupos de trabajo (3-5 alumnos/as) permitió un conocimiento pormenorizado del evento a analizar gracias al reparto de tareas intragrupales. Entre otras, esto llevó al alumnado a examinar diferentes agentes de la gestión musical, como el director artístico o el mánager, y del arte, como el comisario/a de exposición. Por su parte, los resultados del reparto de tareas intergrupales facilitaron que el alumnado generase, colectivamente, un mapa general de los eventos musicales a nivel nacional que se 
desarrollaron durante la temporada. En último término, la elaboración de podcast radiofónicos, haciendo énfasis en el análisis y diagnóstico de exposición o festival musical, y su puesta en común del contenido sonoro en la Muestra Virtual, dio como resultado un material divulgativo destacado, evidenciando la importancia capital de los medios de comunicación en la difusión de actividades culturales.

\subsection{Arqueología del Mundo Clásico (4 Grado en Historia)}

Se realizaron tres vídeos, de los cuales uno se subió a internet por el propio alumnado. Para el profesor, la labor resultó más gratificante de la habitual en comparación con la aplicación de los métodos clásicos de enseñanza. El alumnado se mostró en general implicado con el planteamiento de la asignatura, aunque hubo algún caso concreto en el que no ocurrió así.

Uno de los resultados más destacables fue lograr avances en metodologías docentes innovadoras, principalmente mediante el uso de dos técnicas pedagógicas: el aprendizaje inverso y el aprendizaje a través de experiencias. Ambas fueron nuevas para el profesor y los alumnos, pero demostraron su potencial. Permitieron obtener una serie de beneficios para el alumnado como, por ejemplo, fomentar el aprendizaje activo, practicar el desempeño de su profesión futura y desarrollar competencias importantes en su formación. Entre estas últimas destaca especialmente el poder aplicar los conocimientos adquiridos y su capacidad de resolución de problemas en entornos nuevos o poco conocidos dentro de contextos más amplios (o multidisciplinares) relacionados con su área de estudio.

\subsubsection{Psicología de la Educación y del Desarrollo (1ํㅜ de Grado en Educación Primaria)}

Basándose tanto en el número de trabajos realizados por los distintos grupos, en el número de entradas que se realizaron en los distintos foros y en las respuestas y valoraciones que realizó el alumnado en la encuesta sobre el trabajo de grupo, se considera que la participación y los resultados obtenidos fueron positivos.

En los 3 grupos clase en los que se desarrolló la actividad, se presentaron un total de 44 trabajos, con 720 mensajes en foros, lo que equivale a una media global de 16,3 contribuciones en foros por trabajo. En la tabla 2, aparece esta información diferenciada por grupos.

Tabla 2.

Número de trabajos, de entradas en foros y media de entradas/trabajo por grupo clase.

\begin{tabular}{cccc}
\hline Grupo & Trabajos realizados & $\begin{array}{c}\text { № de entradas totales realizadas } \\
\text { en sus foros }\end{array}$ & $\begin{array}{c}\text { Media de entradas por } \\
\text { trabajo }\end{array}$ \\
\hline 1 & 16 & 188 & 11.75 \\
\hline 2 & 17 & 257 & 25 \\
\hline 4 & 11 & 275 & 16.3 \\
\hline Total & 44 & 720 & \\
\hline
\end{tabular}

Fuente: Elaboración propia. 
Respecto a la muestra virtual realizada, estudiantes de estos grupos incluyen 24 trabajos consistentes en vídeos de en torno a 59 segundos con reflexiones sobre el trabajo realizado. Estos videos generaron 289 entradas en los foros, que supone 12.04 entradas por trabajo.

Como se ha citado, todo el alumnado participante de esta asignatura cumplimentó un cuestionario realizado en una aplicación sobre la valoración que otorgaban a la realización de distintos aspectos del trabajo realizado. En la tabla 3 aparecen las preguntas que se incluyeron en el cuestionario, así como las opciones de respuestas y porcentaje de cada una de las respuestas. Fueron 149 alumnos/alumnas los que contestaron este cuestionario.

Tabla 3.

Preguntas, opciones de respuesta y porcentaje de respuestas incluidas en el cuestionario sobre la valoración del trabajo realizado.

\begin{tabular}{|c|c|c|}
\hline $\begin{array}{c}\text { № de la } \\
\text { Pregunta }\end{array}$ & Enunciado de la pregunta & Opciones de respuesta \\
\hline 1 & ¿Qué te ha aportado realizar esta actividad de grupo? & Varias \\
\hline 2 & $\begin{array}{l}\text { ¿Te hubiera gustado realizar la actividad solo/-a en lugar de } \\
\text { en grupo? }\end{array}$ & $\begin{array}{l}\text { Sí }(13.4 \%) \\
\text { No }(86.6 \%)\end{array}$ \\
\hline 3 & $\begin{array}{l}\text { ¿Cómo consideras el proceso de trabajo y aprendizaje que } \\
\text { habéis llevado a cabo en tu grupo? }\end{array}$ & $\begin{array}{l}\text { Positivo }(87.9 \%) \\
\text { Irregular }(6.7 \%) \\
\text { Negativo }(3.3 \%) \\
\text { Ns/Nc }(2.0 \%)\end{array}$ \\
\hline 4 & $\begin{array}{l}\text { ¿Qué te ha parecido realizar la actividad de grupo } \\
\text { basándose en la aplicación de tecnologías a la docencia? }\end{array}$ & $\begin{array}{l}\text { Positivo }(95.3 \%) \\
\text { Irregular }(1.3 \%) \\
\text { Negativo }(0.6 \%) \\
\text { Ns/Nc }(2.7 \%)\end{array}$ \\
\hline 5 & $\begin{array}{l}\text { ¿Crees que utilizarías alguna de las aplicaciones en la } \\
\text { docencia como maestro o maestra? }\end{array}$ & $\begin{array}{l}\text { Sí (95.3\%) } \\
\text { No }(2.0 \%) \\
\text { Ns/Nc }(2.7) \\
\end{array}$ \\
\hline 6 & Valora el trabajo realizado por tu grupo & $\begin{array}{l}\text { Muy Bueno }(40.9 \%) \\
\text { Bueno }(51.67 \%) \\
\text { Regular }(4.7 \%) \\
\text { Malo }(0 \%) \\
\text { Muy Malo }(0.6 \%) \\
\text { Ns/Nc }(2.0 \%)\end{array}$ \\
\hline 7 & Valora tu trabajo en el grupo & $\begin{array}{l}\text { Muy Bueno (43.0\%) } \\
\text { Bueno }(51.0 \%) \\
\text { Regular }(3.3 \%) \\
\text { Malo }(0 \%) \\
\text { Muy Malo }(0.6 . \%) \\
\text { Ns/Nc }(2.0 \%) \\
\end{array}$ \\
\hline
\end{tabular}

Fuente: Elaboración propia. 
La primera pregunta fue una pregunta abierta que se categorizó en ocho categorías de respuesta no excluyentes, de forma que un alumnado pudo seleccionar más de una. Las categorías y los porcentajes del alumnado fueron: 1) Fomentar la relación con los compañeros/as (16.1\%), 2) Aprender algo respecto al trabajo en grupo $(49.7 \%), 3)$ Obtener más conocimientos (49.7.\%), 4) Mejorar el desempeño $(2.7 \%), 5)$ Conocer otros puntos de vista interesantes $(14.8 \%), 6)$ Aprender el uso de una nueva tecnología $(22.1 \%), 7)$ Se han generado problemas en el grupo $(2.0 \%)$ y 8$)$ Aumento de la motivación de alguna forma (7.4\%).

Como puede observarse en esta primera pregunta y en el resto, en líneas generales los resultados son positivos; siendo casi unánime las ventajas que otorgan al trabajo en grupo con la utilización de TIC. No obstante, aunque la proporción de respuestas que señalan algún aspecto negativo es baja, son una valiosa fuente para detectar qué posibles aspectos no han funcionado adecuadamente, los cuales fueron aquellos principalmente relacionados con el funcionamiento de los grupos y no tanto con la temática y metodología elegida.

Nos gustaría destacar que, en general, hubo una valoración mayoritaria positiva de la utilización de los recursos tecnológicos seleccionados y de la perspectiva de utilizarlos profesionalmente en un futuro.

\subsubsection{Observación Sistemática en el aula de Educación Infantil (1ํ de Grado en Educación Infantil):}

Partir del compromiso negociado aumentó la motivación al trabajo y facilitó la comunicación y el diálogo compartido. En los grupos se manifestó una mayor asunción de responsabilidades por parte de sus miembros, en buena medida debido a que las metas fueron elaboradas por el propio grupo, y al hecho de una mayor sistematicidad en las pautas de organización del trabajo. Los frutos del trabajo de estos contenidos con esta metodología pueden observarse en relación a los resultados académicos obtenidos por el alumnado. El 18,87\% aprobaron; el 39,62\% obtuvieron la calificación de Notable y el 6,77\% consiguieron una nota académica de Sobresaliente o Matrícula de Honor.

\subsection{Actividad: I Muestra Virtual "El aprendizaje cooperativo en 59 segundos"}

Aunque no todo el alumnado de las distintas asignaturas participó de igual manera, durante la muestra virtual hubo participación significativa de alumnado. Se subieron un total de 29 trabajos de grupo, lo que implicó una participación de en torno a 130 alumnos. Se generaron un total de 327 mensajes en los foros, lo que supuso por término medio 11.27 mensajes por trabajo; una buena cifra respecto al intercambio de ideas sobre el trabajo cooperativo en combinación con la utilización de las TIC.

El alumnado que participó en la muestra virtual recibió una acreditación de participación, la cual aparece en las figuras 2 y 3 . Se realizó al objeto de reconocer a aquel alumnado que además de realizar la actividad propia de la asignatura, realizó ra una reflexión sobre la metodología y estrategia de aprendizaje llevada a cabo.

El desarrollo de la muestra permitió que los grupos comentaran aspectos significativos sobre el trabajo cooperativo, incorporando tanto beneficios como potenciales dificultades y desafíos (García-Valcárcel y Tejedor, 2017), favoreciendo el enriquecimiento por parte de todos los participantes en el proyecto (profesorado y alumnado). Por un lado, la creación de este espacio virtual sirvió al alumnado participante para compartir impresiones y vivencias vinculadas al uso de las TIC como instrumento. Por otro lado, la habilitación foros de trabajo en los que debatir y 
reflexionar sobre las TIC y el trabajo cooperativo, permitió valorar como el alumnado utilizó las herramientas tecnológicas para debatir, exponer y reflexionar sobre cómo aprenden con ellas. La diversidad de propuestas llevadas a cabo en las diferentes asignaturas y grados mostró los dos elementos articuladores del proyecto: el empleo de la metodología cooperativa y el uso de las TIC.

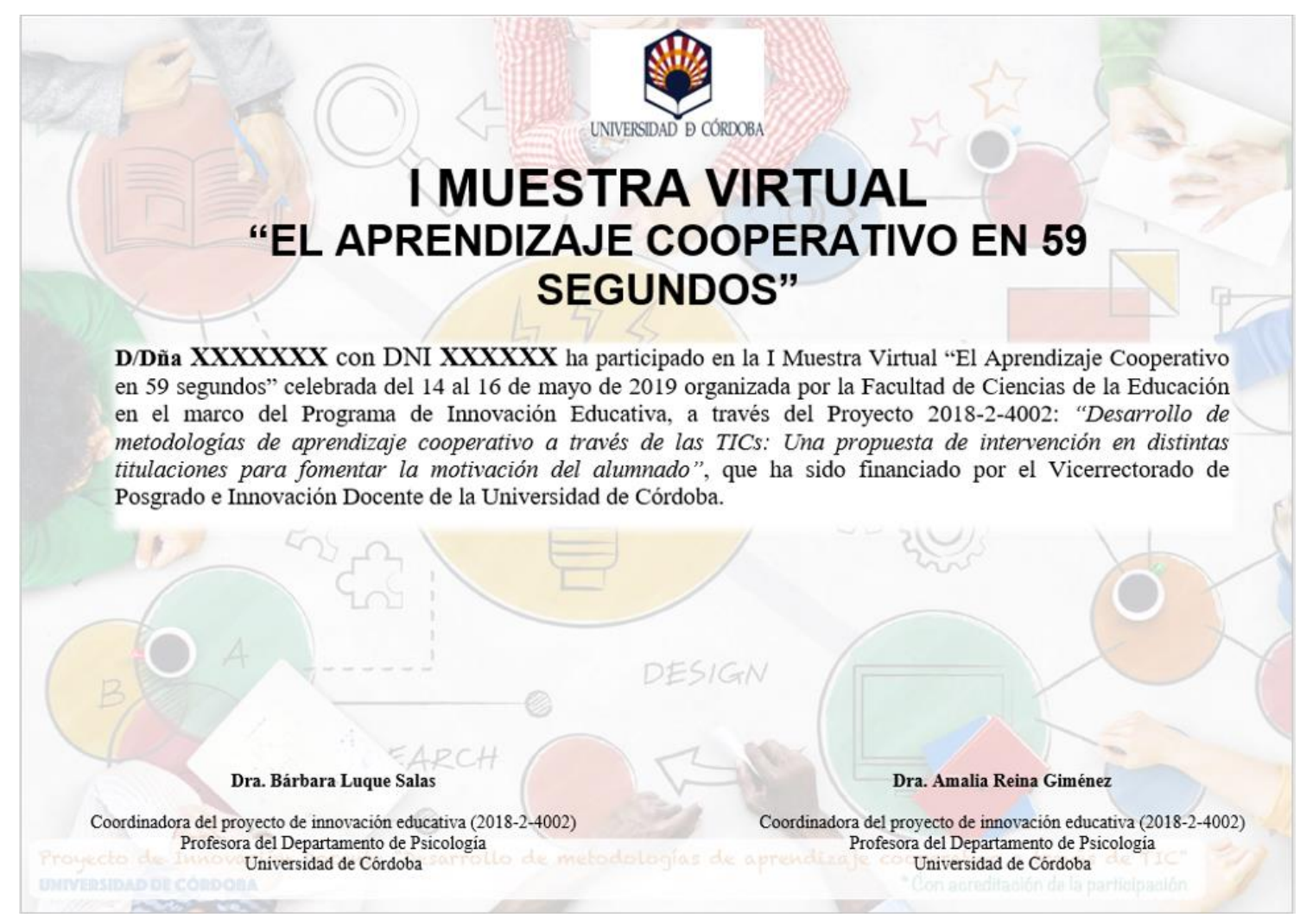

Figura 2. Acreditación de participación en la muestra. 


\section{MUESTRA VIRTUAL “EL APRENDIZAJE COOPERATIVO EN 59 SEGUNDOS”}

Esta actividad se desarrolla para fomentar la transferencia y el intercambio de conocimientos y experiencias del alumnado. El objetivo de la misma ha sido crear un espacio virtual en el que compartir impresiones y vivencias vinculadas al intercambio dialógico entre estudiantes, bajo la supervisión y orientación de los docentes.

A partir de la realización de trabajos/proyectos de grupo previos en asignaturas de distintos Grados y grupos con el común denominador del uso de TICs, durante la muestra se han habilitado foros de trabajo en los que debatir y reflexionar sobre las potencialidades y limitaciones del trabajo cooperativo y sus características.

La participación, ha consistido de un parte en mostrar cada grupo en un vídeo de unos 59 segundos alguna reflexión o aspecto sobre el trabajo cooperativo que se ha abordado. De otra parte los participantes han visionado distintos vídeos de otros grupos para su posterior análisis y por último, han realizado participaciones en forma de conversación en los diferentes foros, tanto sobre los videos visualizados y como las aportaciones recibidas. Durante su desarrollo se ha conseguido que los grupos comenten aspectos significativos sobre el trabajo cooperativo, incorporando tanto beneficios como potenciales dificultades y desafíos.

Equipo del proyecto de innovación educativa (2018-2-4002)

Figura 3. Reverso de la acreditación de participación en la muestra.

\section{CONCLUSIONES}

Se considera que la valoración de los resultados alcanzados permite afirmar que se consiguieron distintos objetivos específicos, tales como:

- Lograr una mayor formación de los docentes implicados en el uso de una metodología alternativa de enseñanza como es el aprendizaje cooperativo.

- Favorecer una mayor implicación en el proceso de enseñanza-aprendizaje tanto del alumnado como del profesorado que ha participado en las actividades.

- Fomentar el desarrollo de las capacidades de investigación autónoma del alumnado, habiendo resultado un elemento tanto de motivación como de introducción a la investigación.

- Favorecer la participación y creatividad del alumnado, mediante la realización de actividades en un formato grupal y cooperativo.

- Desarrollar roles diferenciados por parte del alumnado, principalmente en aquellas asignaturas en las que hubo un reparto de los roles en los grupos de trabajo.

- Incentivar el conocimiento y uso de distintas aplicaciones basadas en las TIC susceptibles de ser utilizadas por el alumnado en distintos ámbitos de actuación profesionales.

No obstante, aún con la consecución de estos objetivos, una revisión de las conclusiones que se pueden derivar de los mismos, de las limitaciones que han podido 
surgir y de posibles aspectos de mejora, llevan al análisis de distintos aspectos que a continuación se detallan y que es positivo tener en cuenta:

Ratio profesorado/alumnado: una elevada ratio de estudiantes con respecto a los docentes supone una dificultad para la realización de las actividades que han formado parte del proyecto de innovación que se desarrolló y aquí se ha presentado. Para el desarrollo de actividades de este tipo, es conveniente contar con una ratio de alumnado/profesorado inferior, siendo el formato más adecuado la realización en clases prácticas en las que el número de grupos por profesor/a esté en torno a 4-5, siendo similar el número de alumnos/as por grupo. Hay que tener en cuenta que aunque el trabajo cooperativo combinado con la utilización de las TIC puede facilitar la consecución de objetivos como los citados, también necesita de una atención por parte del profesorado precisa y adecuada, la cual se torna como más importante en los momentos iniciales y hasta que el grupo adquiere una autonomía adecuada.

Contexto de las actividades: habida cuenta de que todo el curso académico está igualado en cuanto a carga de trabajo para el estudiantado, en los periodos de más carga docente -fundamentalmente en época final del cuatrimestre o en época de exámenes- el alumnado puede responder de un modo menos efectivo a estas actividades.

Puede ocurrir y a veces ocurre, que el alumnado da más prioridad o tiene más tendencia a preparar asignaturas basadas en los métodos tradicionales de enseñanza, en comparación con asignaturas que se basan en metodologías innovadoras; las que en cierta medida le pueden suponer una dedicación diferente a la habitual bien en cantidad o en calidad, lo cual puede generar cierta resistencia.

El hacer consciente al alumnado de la importancia de metodologías diversas; así como tener en cuenta el calendario del alumnado para evitar posibles coincidencias y sobrecargas, son elementos importantes para favorecer la motivación e implicación que puede encontrarse con actividades de este tipo y efectivamente aprovechar la oportunidad que suponen. Además, aunque pueda ser debatible su pertinencia o no, se entiende como recomendable que todas las actividades tengan un reflejo en la calificación; de lo contrario la participación del estudiantado puede ser baja.

Seguimiento sistemático: se considera que no sólo es necesario explicar detalladamente la manera de abordar el trabajo, sino que el profesorado debe ser sistemático en el seguimiento. En este sentido, es de importancia que se recuerde en cada sesión cómo se comienza y cómo se termina ese bloque de trabajo y se vaya monitorizando la progresión del proceso de aprendizaje con el alumnado en función de las actividades programadas.

Autonomía: para la realización de actividades cooperativas de forma óptima es necesario lograr la autonomía del alumnado, desde el punto de vista de su capacidad para el autoaprendizaje. Esto requiere de una orientación y supervisión precisa y continua en el tiempo por parte del profesorado. Conforme el grupo de estudiantes consigue realizar la dinámica de forma autónoma -lo que suele llevar tiempo, dado los sesgos previos y el proceso de aprendizaje en sí mismo- el profesorado podrá ir dando mayor libertad a los y las estudiantes. Sin la consecución de este objetivo en sí mismo, 
el trabajo en grupo corre el riesgo de convertirse una suma de aportaciones individuales sin una visión integradora común y con un aprovechamiento limitado.

\section{REFERENCIAS}

Colomina, R. y Onrubia, J. (2001). Interacción educativa y aprendizaje escolar: la interacción entre alumnos. En C. Coll, J. Palacios y A. Marchesi (Comps.): Desarrollo psicológico y educación, II: Psicología de la educación escolar. Madrid: Alianza.

Fernández Berrocal, P. y Melero, M.A. (Comps). (1995). La interacción social en contextos educativos. Madrid: Siglo XXI.

García-Valcárcel, A. y Tejedor, F.J. (2017). Percepción de los estudiantes sobre el valor de las TIC en sus estrategias de aprendizaje y su relación con el rendimiento. Educación XX1, 20 (2). 137-159.

García Pérez, F. F. (2000). Un modelo didáctico alternativo para transformar la educación: el Modelo de Investigación en la Escuela. Scripta nova. Revista Electrónica de Geografía y Ciencias Sociales, 4 (64), 1-24.

García Díaz, J.E. y García Pérez, F.F (1989): Aprender investigando. Una propuesta metodológica basada en la investigación. Sevilla. Diada

Herrada, R.I. y Baños, R. (2018). Aprendizaje cooperativo a través de las nuevas tecnologías: Una revisión. @tic: Revista d'Innovació Educativa, 20, 16-25

La Cueva, A. (1989) La enseñanza por proyectos: ¿mito o reto?, Revista Iberoamericana de Educación 16, 1998, 165-187.

Ortega, J., y Sánchez-Quejia, I. (2014): Variables psicológicas implicadas en el aprendizaje: el sentido del aprendizaje. En Prados, M.M., Sánchez, V., Sánchez-Quejia, I., Del Rey, R., Pertegal, M.A., Reina, M.C., Ridao, P., Ortega, F.J. y Mora, J. Manual de psicología de la educación para docentes de Educación Infantil y Primaria. (pp. 85-105). Madrid. Pirámide.

Palomares-Montero, D. y Chisvert-Tarazona, M. J. (2016). Cooperative learning: a methodological innovation in teacher training/El aprendizaje cooperativo: una innovación metodológica en la formación del profesorado. Cultura y Educación, 28(2), 378-395. doi: 10.1080/11356405.2016.1158448

Pineda, J. A. y García, F. (2011): La construcción de un ámbito de investigación escolar sobre el conflicto, la violencia y la guerra, íber. Didáctica de las Ciencias Sociales, Geografía e Historia 68, 2011, 82-91 